\title{
KR-31831, benzopyran derivative, inhibits VEGF-induced angiogenesis of HUVECs through suppressing KDR expression
}

\author{
SHI-YOUNG PARK ${ }^{1}$, EUN-HEE SEO ${ }^{1}$, HYUN SEOK SONG ${ }^{1}$, SEUNG-YOUN JUNG ${ }^{1}$, \\ YOUNG-KYOUNG LEE ${ }^{1}$, KYU-YANG YI ${ }^{2}$, SUNG-EUN YOO ${ }^{2}$ and YUNG-JIN KIM $^{1}$ \\ ${ }^{1}$ Department of Molecular Biology, Pusan National University, Busan 609-735; \\ ${ }^{2}$ Korea Research Institute of Chemical Technology, Daejeon 305-600, Korea
}

Received December 27, 2007; Accepted February 5, 2008

\begin{abstract}
Angiogenesis is important in the development and progression of cancer, therefore the therapeutic approach based on anti-angiogenesis may represent a promising therapeutic option. KR-31831 is a novel anti-ischemic agent. Previously, we reported the anti-angiogenic activity of KR-31831. In the present study we investigated the molecular mechanisms underlying anti-angiogenic activity of KR-31831. We show that KR-31831 inhibits vascular endothelial growth factor (VEGF)-induced proliferation and tube formation via release of intracellular $\mathrm{Ca}^{2+}$ and phosphorylation of extra-cellular regulated kinase $1 / 2($ Erk1/2) in human umbilical vein endothelial cells (HUVECs). Moreover, the expression of VEGF receptor 2 (VEGFR2, known as Flk-1 or KDR) was reduced by the treatment of KR-31831. These results suggest that KR-31831 may have inhibitory effects on tumor angiogenesis through down-regulation of KDR expression.
\end{abstract}

\section{Introduction}

Angiogenesis is the formation of new blood vessels from pre-existing vessels that occurs in many physiological and pathological conditions such as embryonic development, chronic inflammation, wound healing, and tumor progression and metastasis. Angiogenesis is tightly controlled by a wide variety of regulators which include growth factors, cytokines, lipid metabolites and cryptic fragments of hemostatic proteins (1). VEGF is a potent angiogenic stimulator produced by

Correspondence to: Dr Yung-Jin Kim, Department of Molecular Biology, Pusan National University, Busan 609-735, Korea E-mail: yjinkim@pusan.ac.kr

Abbreviations: VEGF, vascular endothelial cell growth factor; KDR, kinase insert domain-containing receptor; HUVECs, human umbilical vein endothelial cells; Erk1/2, extracellular regulated kinase $1 / 2$

Key words: angiogenesis, anti-angiogenesis, KR-31831, VEGF, VEGFR2 various types of tumor cells, which plays an important role in the growth and metastasis of tumors by promoting neovascularization (2-5).

Angiogenic activity of VEGF is thought to be mediated by the high-affinity receptor tyrosine kinases, VEGFR 1 ( $f m s$-like tyrosine kinase, Flt-1) and VEGFR2 (kinase insert-domain containing receptor, KDR, in human and its murine homologue, fetal liver kinase-1, Flk-1). Although both receptors are co-expressed on most endothelial cells VEGFR-2 is thought to dominate the angiogenic response Binding of VEGF to its receptors causes dimerization of the receptor and activation of the intrinsic tyrosine kinase domain, followed by autophosphorylation of the receptor and subsequent signal transduction.

KR-31831 (2R,3R,4S)-6-amino-4-[n-(4-chlorophenyl)-N(1H-imidazol-2ylmethyl)amino]-3-hydroxyl-2-methyl-2dimethoxymethyl-3,4-dihydro-2H-1-benzopyran, is a novel synthetic anti-ischemic agent. Benzopyran is one of the most frequently used back-bones of synthetic drugs, including anti-oxidants, anti-hypertensive and therapeutic agents for ischemia-related diseases. A variety of amines were introduced at the 4-position of benzopyran for the identification of ATP sensitive potassium channel $\left(K_{\text {ATP }}\right)$ openers targeting ischemic diseases, such as myocardial infarction and stroke (6). Previously, we reported that KR-31831 plays a role as a novel anti-angiogenic agent in bovine aortic endothelial cells (BAECs) (7). KR-31831 suppressed endothelial cell proliferation, tube formation, invasion and migration in vitro. Also, this agent inhibited vessel formation in the mouse Matrigel plug assay in vivo.

In the present study we investigated the inhibitory mechanism of KR-31831 on tumor angiogenesis, especially on the VEGF-signaling pathway in HUVECs. Our data demonstrate that KR-31831 down-regulated VEGF-induced tumor formation and proliferation of HUVECs by inhibiting intracellular $\mathrm{Ca}^{2+}$ release and Erk1/2 activation. These findings will broaden our understanding of the molecular mechanisms of KR-31831 as a novel anti-angiogenic agent.

\section{Materials and methods}

Materials. KR-31831 was obtained from Korea Research Institute of Chemical Technology. Matrigel was purchased 
from BD Biosciences (Bedford, MA). VEGF, ionomycin, gelatin (2\%), SU-5416 and protease inhibitor cocktail were purchased from Sigma Chemical Co. (St. Louis, MO). Antibodies for Erk1/2, phospho-Erk1/2 and KDR were purchased from Cell Signaling Technology (Danvers, MA). Goat antirabbit IgG-HRP and anti-mouse IgG-HRP were purchased from Santa Cruz Biotechnology (Santa Cruz, CA).

Cell culture. HUVECs (Cambrex, Charles City, IA) were grown according to the manufacturer's protocol in EBM-2 medium (Cambrex, Charles City, IA) supplemented with EGM-2 kit containing fetal calf serum (FCS, 2\%), hydrocortisone $(0.04 \%)$, hFGF-B $(0.4 \%)$, VEGF $(0.1 \%)$ and heparin $(0.1 \%)$. Cells were incubated in a humidified atmosphere of $5 \% \mathrm{CO}_{2}$ in air at $37^{\circ} \mathrm{C}$. HUVECs in passages 5 through 8 were used in all experiments.

Cell proliferation. The effect of KR-31831 on proliferation of HUVECs was examined by MTT (3-(4,5-dimethylthiazolyl-2)2,5-diphenyltetrazolium bromide) assay. HUVECs were grown in EGM-2 medium at a density of $1 \times 10^{4}$ cells/well on 24-well plates. After overnight incubation, the medium was changed with EBM-2 containing $1 \%$ of fetal bovine serum (FBS) and incubated for 3 days. MTT solution $(5 \mathrm{mg} / \mathrm{ml}$ dissolved in $\mathrm{H}_{2} \mathrm{O}$ ) was then added to the well to a final concentration of $0.5 \mathrm{mg} / \mathrm{ml}$. After a 4-h incubation, $0.3 \mathrm{ml}$ of dimethyl sulfoxide (DMSO) was added to the wells to dissolve the MTT-formazan. All samples were assayed in triplicate and independently repeated three times.

Tube formation assay. HUVECs $\left(5 \times 10^{5}\right.$ cells/well) were seeded on a layer of polymerized Matrigel. KR-31831 $(0.1 \mu \mathrm{M})$ was added to each well with or without VEGF (100 ng/ml) and the cells were incubated at $37^{\circ} \mathrm{C}$. After $12 \mathrm{~h}$ change in cell morphology were observed under a phase-contrast microscope and photographed. The effect of KR-31831 was compared to that of SU-5416 (an antagonist of VEGF receptor; $20 \mu \mathrm{M}$ ).

Immunoblot analysis. Cells were lysed with ice-cold extraction buffer [50 mM Tris- $\mathrm{HCl}$ (pH 8.0), $150 \mathrm{mM} \mathrm{NaCl}, 1 \% \mathrm{NP}-40$, $0.1 \%$ SDS, $0.5 \%$ sodium deoxycholate] containing a protease inhibitor cocktail. Protein in lysate was quantitated using the Bradford method (and bovine serum albumin as a standard), resolved over a 6 or $10 \%$ sodium dodecylsulfate-polyacrylamide gel using electrophoresis (SDS-PAGE), and electroblotted to a polyvidyline fluoride (PVDF) membrane (Hybond-P; Amersham Pharmacia Biotech, Ltd., Bucks., $\mathrm{UK})$. Membrane with protein was blocked for $2 \mathrm{~h}$ at room temperature in TBS containing $0.1 \%$ Tween-20 and 5\% skim milk, and then incubated with antibodies against phosphoErk1/2 (1:1000), Erk1/2 (1:2000), KDR (1:1000) and $\alpha$-tubulin (1:5000). Peroxidase-conjugated anti-rabbit or anti-mouse secondary antibody was used at a dilution of 1:2000 or 1:5000. Signals were visualized using the West One ${ }^{\mathrm{TM}}$ Western Blot Detection System (iNtRON Biotechnology, Korea). Each assay was performed in triplicate.

RT-PCR analysis. Total RNA from HUVECs was isolated according to the manufacturer's instructions using easy-Blue ${ }^{\mathrm{TM}}$ Total RNA Extraction Kit (iNtRON Biotechnology). cDNA was synthesized by M-MLV reverse transcriptase (Promega Co., Madison, WI) with $2 \mu \mathrm{g}$ of total RNA and oligo-(dT)15 (Life Technologies, NY). Equal amounts of cDNA were subjected to PCR amplification using PCR premix kit (Maxime PCR PreMix (i-Taq), iNtRON Biotechnology) and gene-specific primers: KDR, 5'-GCA TCT CAT CTG TTA CAG C-3' (forward) and 5'-CTT CAT CAA TCT TTA CCC C-3' (reverse); ß-actin, 5'-GAC TAC CTC ATG AAG ATC3' (forward) and 5'-GAT CCA CAT CTG CTG GAA-3' (reverse). The PCR products were electophoresed on $1 \%$ agarose gels and visualized by ethidium bromide staining under UV trans-illumination.

Cell cycle analysis. After fixation with $80 \%$ ethanol for $10 \mathrm{~min}$ at $4^{\circ} \mathrm{C}$, HUVECs were incubated with RNase for $30 \mathrm{~min}$ at $37^{\circ} \mathrm{C}$ and then stained with propidium iodide (PI) for $5 \mathrm{~min}$. Flow cytometer (FC500, Beckman Coulter, Fullerton, CA) was used for cell cycle analysis. The cell cycle was separated into three different phase, $\mathrm{G}_{0} / \mathrm{G}_{1}, \mathrm{~S}$ and $\mathrm{G}_{2} / \mathrm{M}$ based on DNA content. After carefully identifying and gating cell populations for the respective phases, we determined the percentage of cells in the different phases from subjectively applied markers in the DNA histogram. Assay was performed in triplicate.

Data analysis. Results were expressed as the mean \pm SD. The statistical differences among the results of the various groups were compared by the Student's t-test. A $p<0.05$ was considered significant.

\section{Results}

KR-31831 inhibits VEGF-induced tube formation and proliferation of HUVECs. Previously, we reported that KR-31831 inhibits in vivo and in vitro angiogenesis of BAECs (7). We tested the activity of KR-31831, a benzopyran derivative (Fig. 1A), on VEGF-induced angiogenesis in HUVECs. HUVECs were placed on a Matrigel-coated plate and incubated for $12 \mathrm{~h}$. HUVECs on Matrigel formed blood vessel networks induced by VEGF, whereas KR-31831 (10 $\mu \mathrm{M})$ inhibited VEGF-induced tube formation of HUVECs (Fig. 1B). The proliferation of endothelial cells is one of the critical features in the formation of new blood vessels (8). To determine the effect of KR-31831 on cell proliferation we performed an MTT assay. As shown in Fig. 1C, treatment with KR-31831 inhibited proliferation of HUVECs after $48 \mathrm{~h}$. At $72 \mathrm{~h}$, treatment with KR-31831 $(10 \mu \mathrm{M})$ inhibited the proliferation of HUVECs by approximately half relative to the proliferation of controls. These results indicate that KR-31831 exerts antiangiogenic activity through inhibition of tube formation and proliferation.

KR-31831 down-regulates VEGF-induced activation of ERK1/2 pathway and increases intracellular $\mathrm{Ca}^{2+}$. To understand the molecular mechanism of the inhibition of VEGFinduced angiogenesis by KR-31831, we investigated VEGF signaling. VEGF activates proliferation, survival, migration and invasion of endothelial cells, and permeability of blood vessels through VEGF receptors, especially KDR (9). KDR activated by binding to VEGF induces cellular proliferation through activation of the classic Erk1/2 pathway, which 
A<smiles>COC(OC)[C@]1(O)Oc2ccc(N)cc2[C@@H]1N(Cc1ncc[nH]1)c1ccc(Cl)cc1</smiles>

B

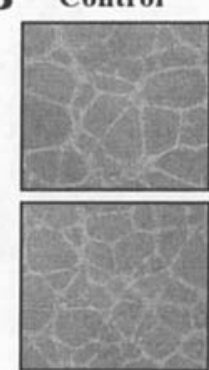

VEGF

+ KR-31831

C

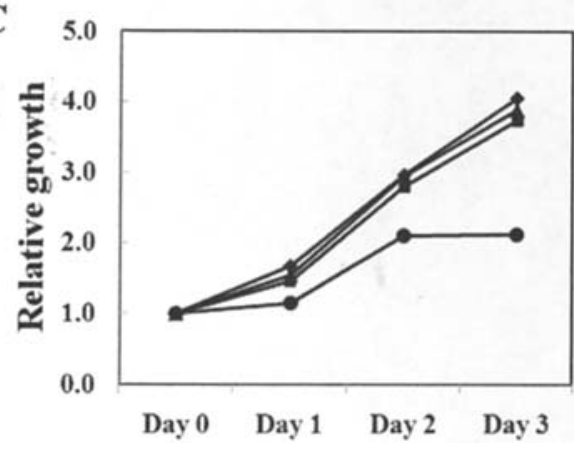

VEGF

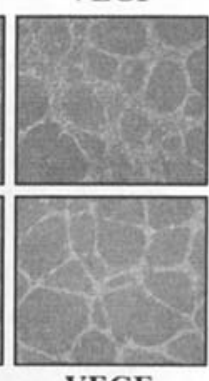

VEGF

+ SU 5416

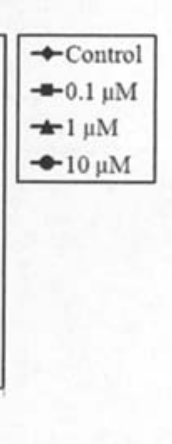

Figure 1. KR-31831 inhibits VEGF-induced tube formation and proliferation of HUVECs. A, The structure of KR-31831. B, HUVECs $\left(5 \times 10^{5}\right.$ cells) were seeded on a layer of polymerized Matrigel. Cells were treated with KR-31831 $(10 \mu \mathrm{M})$ after VEGF-treatment $(10 \mathrm{ng} / \mathrm{ml})$ and incubated at $37^{\circ} \mathrm{C}$ for $12 \mathrm{~h}$. SU-5416 $(20 \mu \mathrm{M})$ was used as a positive control for anti-angiogenesis. Changes of cell morphology were examined under a phase-contrast microscope and photographed. C, Starved HUVECs ( $1 \times 10^{4}$ cells) were treated with VEGF $(10 \mathrm{ng} / \mathrm{ml})$ alone or with KR-31831 $(0.1,1$ and $10 \mu \mathrm{M})$. After 3 days an MTT assay was performed to determine the proliferation of HUVECs. All samples were assayed in triplicate and repeated independently three times.

leads to gene expression (10). Thus, we performed Western blot analysis to determine the effect of KR-31831 on VEGFinduced phosphorylation of Erk1/2. Starved HUVECs were incubated in the presence or absence of KR-31831 $(0.1,1$ or $10 \mu \mathrm{M})$ for $24 \mathrm{~h}$, and then, stimulated with VEGF (10 ng/ml) for $10 \mathrm{~min}$. The result indicates that VEGF-induced Erk1/2 activation was significantly inhibited by $10 \mu \mathrm{M}$ KR-31831, while a small decrease in Erk1/2 activation was observed with 0.1 and $1 \mu \mathrm{M}$ KR-31831 (Fig. 2A).

It has also been reported that activation of Erk1/2 by KDR is mediated by the protein kinase $\mathrm{C}$ (PKC) signal pathway (20). Phospholipase C- $\gamma$ (PLC- $\gamma$ ) activation by KDR leads to generation of the second messengers, $s n$-1,2-diacyl-glycerol (DAG) and inositol (1,4,5)-triphosphate $\left(\mathrm{IP}_{3}\right)$. PKC is activated by DAG and increased levels of intracellular $\mathrm{Ca}^{2+}$ induced by $\mathrm{IP}_{3}$. Increase in intracellular $\mathrm{Ca}^{2+}$ concentration is also key to activate Erk1/2 along the VEGF signaling pathway. Therefore, we investigated the effect of KR-31831 on $\mathrm{Ca}^{2+}$ signal using ionomycin, a $\mathrm{Ca}^{2+}$ ionophore which increases intracellular $\mathrm{Ca}^{2+}$ level. Serum starved HUVECs were incubated in the presence or absence of KR-31831 $(10 \mu \mathrm{M})$ for $24 \mathrm{~h}$ and then stimulated with VEGF $(10 \mathrm{ng} / \mathrm{ml})$ and ionomycin $(200 \mu \mathrm{M})$ for $10 \mathrm{~min}$. The ionomycin treatment reversed the decrease of Erk1/2 activation by KR-31831 (Fig. 2B). These results show that KR-31831 inhibits the increase of intracellular $\mathrm{Ca}^{2+}$ levels and down-regulates the phosphorylation of Erk1/2.
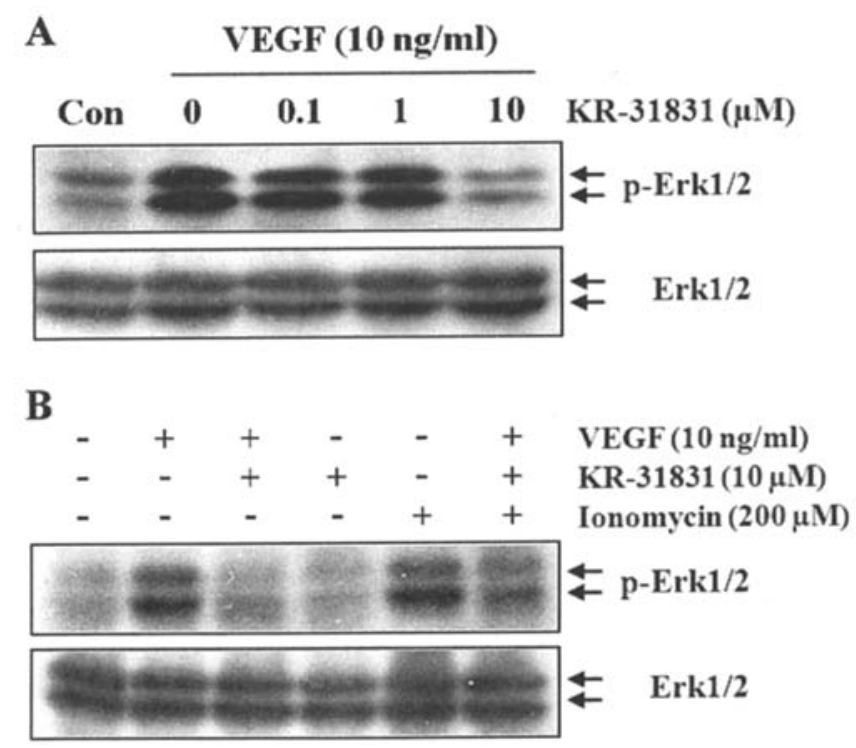

Figure 2. KR-31831 down-regulates phosphorylation of Erk1/2 and intracellular $\mathrm{Ca}^{2+}$ signaling. A, HUVECs were treated with various concentrations of KR-31831 $(0.1,1$ and $10 \mu \mathrm{M})$ for $24 \mathrm{~h}$ and then stimulated with VEGF $(10 \mathrm{ng} / \mathrm{ml})$ for $10 \mathrm{~min}$. Phosphorylation levels of Erk1/2 were examined by Western blot analysis. B, HUVECs were treated with KR-31831 $(10 \mu \mathrm{M})$ and ionomycin $(200 \mu \mathrm{M})$ before stimulation with VEGF (10 ng/ml). Phosphorylation levels of Erk1/2 were examined by Western blot analysis.
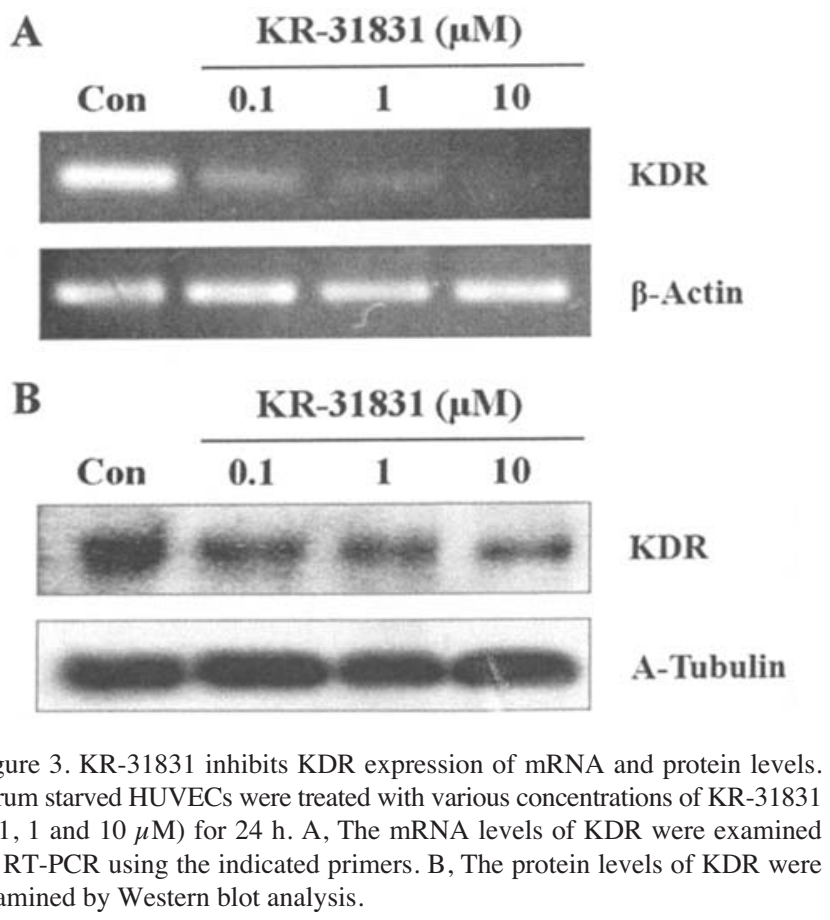

$K R-31831$ reduces the expression of $K D R$ in both $m R N A$ and protein levels. To examine whether the inhibition of both $\mathrm{Ca}^{2+}$ release and Erk1/2 activation by VEGF result from the effect of KR-31831 on KDR we performed RT-PCR and Western blot analysis of KDR expression. Serum starved HUVECs were incubated in the presence or absence of KR-31831 $(0.1,1$ and $10 \mu \mathrm{M})$ for $24 \mathrm{~h}$. KDR mRNA and protein levels were down-regulated by KR-31831 in a dosedependent manner (Fig. 3) and KDR expression was strongly inhibited by $10 \mu \mathrm{M}$ KR-31831. 


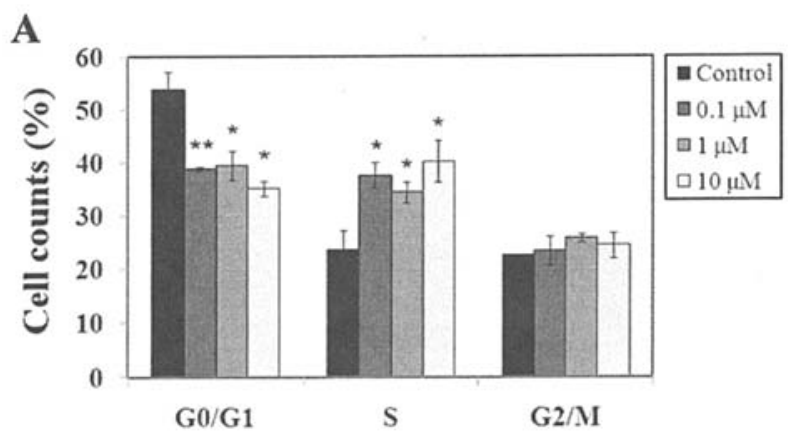

B

\begin{tabular}{|c|c|c|c|c|c|c|c|}
\hline KR-31\$31 $(\mu \mathrm{M})$ & Control & & 1 & & 1 & & 10 \\
\hline $\mathrm{G} 0 / \mathrm{G} 1$ & $53.82 \pm 3.29$ & 38.83 & \pm 0.31 & 39.57 & \pm 2.69 & 35.24 & $\pm 1.3 \mathrm{~s}$ \\
\hline S & $23.65 \pm 3.56$ & 37.62 & \pm 2.38 & 34.51 & \pm 1.91 & 40.30 & \pm 3.85 \\
\hline $\mathrm{G} 2 / \mathrm{M}$ & $22.53 \pm 0.27$ & 23.55 & \pm 2.69 & 25.94 & \pm 0.81 & 24.55 & \pm 2.38 \\
\hline
\end{tabular}

Figure 4. KR-31831 arrests cell cycle of HUVECs in the S-phase. HUVECs was treated with KR-31831 at various concentrations $(0.1,1$ and $10 \mu \mathrm{M})$ and incubated for $24 \mathrm{~h}$. Cell cycles were monitored by flow cytometer. A, The graph represents the percentage of cells in each stage. Each bar represents the mean $( \pm \mathrm{SD})$ of three experiments. ${ }^{*} \mathrm{P}<0.001$ versus control. ${ }^{* *} \mathrm{P}<0.05$ versus control. B, The mean values $( \pm \mathrm{SD})$ are presented.

KR-31831 arrests the cell cycle of HUVECs in the S-phase. The observed inhibitory effects of KR-31831 on VEGF-induced angiogenesis through decreased intracellular $\mathrm{Ca}^{2+}$ signaling, phosphorylation of Erk1/2, and KDR expression raised the possibility that KR-31831 may arrest the HUVEC cell cycle. Because the VEGF signaling pathway, including the ERK1/2 pathway, is essential for the proliferation of endothelial cells we examined whether KR-31831 arrests the cell cycle of HUVECs by incubating HUVECs in the presence or absence of KR-31831 $(0.1,1$ and $10 \mu \mathrm{M})$ for $24 \mathrm{~h}$ and assessing growth arrest by staining the cells with propidium iodide (PI) and analyzing them in a flow cytometer. As shown in Fig. 4, the number of HUVECs in the $\mathrm{G}_{0} / \mathrm{G}_{1}$ phase decreased $15-18 \%$ of control under all KR-31831 concentrations used. In contrast, progression into the S-phase increased $14-17 \%$ of control under all KR-31831 concentrations used. The percentage of HUVECs in G2/M phase was not significantly changed by KR-31831 treatment. This result means that KR-31831 inhibits cell cycle of HUVECs in S-phase.

\section{Discussion}

Angiogenesis is critical in primary tumor growth and metastasis. Over 30 years ago it was reported that tumors were unable to grow beyond 2-3 $\mathrm{mm}$ in the absence of a new vascular supply (2). The importance of angiogenesis to tumor growth provided the rationale for exploring anti-angiogenic therapies in the treatment of cancer (11). Anti-angiogenic drugs in development can be categorized by mechanisms of action: agents that block degradation of extracellular matrix, e.g. matrix metalloproteinase inhibitors; drugs that directly inhibit endothelial cell proliferation and/or migration; agents that inhibit endothelial cell-specific integrin/survival signaling; agents that block promoters of pro-angiogenic genes, e.g. anti-VEGF agents; and drugs with unknown mechanisms of action. However, some of these agents when tested alone or in combination with chemotherapy have failed to show clinical efficacy. Recently, the category of drugs which have shown the most promise are those targeting VEGF signaling (12). Because VEGF and its receptors play a critical role in angiogenesis and tumor progression, many approaches have been developed to inhibit this pathway. These include the development of: a) neutralizing antibodies and soluble receptors that inhibit the binding of VEGF to its receptors, b) antisense constructs against VEGF mRNA, c) mammalian target of rapamycin inhibitors, d) hypoxia inducible factor (HIF) antagonists and e) tyrosine kinase inhibitors that block downstream signaling from membrane-bound VEGF receptors. Based on these concepts, various KDR inhibitors including receptor-specific antibodies and low-molecularweight chemicals such as PTK787/ZK222584, AZD6474 and SU11248, have recently been developed $(13,14)$. Clearly, these small molecules have the potential for treatment of cancers and their metastasis. However most of these agents are in early clinical development.

Previously, we proved that KR-31831 has anti-angiogenic activity (7). KR-31831 inhibited the proliferation, migration, invasion and tube formation of bovine aortic endothelial cells (BAECs) and suppressed in vivo angiogenesis in mouse Matrigel assay. In this study, we investigated the molecular mechanism of KR-31831 on VEGF-induced angiogenesis in HUVECs. First we confirmed the inhibitory activity of KR-31831 on angiogenesis. KR-31831 inhibits the VEGFinduced tube formation of HUVECs like SU-5416 (Fig. 1B). SU-5416 (Semaxanib ${ }^{\text {TM }}$, Sugen Inc.) is a small molecular exhibiting potent and selective inhibition of KDR (15). SU-5416 exerted a potent and rapid anti-proliferative effect on endothelial cells in vitro. When administered to mice, it inhibited the growth of tumor cells in a variety of tumor models (16-19). KR-31831 $(10 \mu \mathrm{M})$ also inhibited VEGFinduced proliferation of HUVECs (Fig. 1C). These results indicate that KR-31831 has the capacity of an anti-angiogenic agent.

The next issue that challenged us was how KR-31831 enabled the inhibition of HUVECs proliferation. Physiologically, KDR, which is activated by VEGF, undergoes dimerization and ligand-dependent tyrosine phosphorylation in intact cells and results in a mitogenic, chemotactic and prosurvival signal (20). In endothelial cells, VEGF induces the phosphorylation of several proteins, such as PLC- $\gamma$, phosphoinositide-3 kinase, Ras GTPase-activating protein and the Src family. In addition, VEGF induces endothelial cell growth by activating the Raf-Mek-Erk pathway. Because KR-31831 inhibited VEGF-induced proliferation we examined the effect of KR-31831 on the phosphorylation of Erk1/2, an essential event in VEGF-signaling. Phosphorylation of Erk1/2 induced by VEGF was down-regulated to control level by treatment with KR-31831 (Fig. 2A). Also, intracellular $\mathrm{Ca}^{2+}$ release upstream of Erk1/2 phosphorylation was reduced by KR-31831 (Fig. 2B). This means that the point of action for KR-31831 is upstream of intracellular $\mathrm{Ca}^{2+}$ release and Erk1/2. Therefore, we examined changes in KDR expression levels, which is the start point of VEGF signaling. Both tran- 
scription and translation of KDR were down-regulated by KR-31831 (Fig. 3). This reduction of KDR expression resulted in the inhibition of VEGF-signaling and cell cycle arrest by KR-31831 (Fig. 4). Strong evidence suggests that blocking KDR limits the ability of most tumors to stimulate the formation of blood vessels (21). The data corroborate the fact that inhibition of KDR suppresses angiogenesis. Collectively, our data indicate that KR-31831 might be a potent inhibitor, antagonizing tumor angiogenesis via KDR regulation and may be a useful agent for the treatment of angiogenesis-related diseases.

\section{Acknowledgments}

This work was supported by a grant from the 2004 Korean National Cancer Control Program (0220060-3), Ministry of Health \& Welfare, Korea.

\section{References}

1. Bussolino F, Mantovani A and Persico G: Molecular mechanisms of blood vessel formation. Trends Biochem Sci 22: 251-256, 1997.

2. Folkman J: Tumor angiogenesis: therapeutic implications. $\mathrm{N}$ Engl J Med 285: 1182-1186, 1971.

3. Kim KJ, Li B, Winer J, et al: Inhibition of vascular endothelial growth factor-induced angiogenesis suppresses tumour growth in vivo. Nature 362: 841-844, 1993.

4. Plate KH, Breier G, Weich HA and Risau W: Vascular endothelial growth factor is a potential tumour angiogenesis factor in human gliomas in vivo. Nature 359: 845-848, 1992.

5. Senger DR, Perruzzi CA, Feder J and Dvorak HF: A highly conserved vascular permeability factor secreted by a variety of human and rodent tumor cell lines. Cancer Res 46: 5629-5632, 1986.

6. Yoo SE, Yi KY, Lee S, et al: A novel anti-ischemic ATP-sensitive potassium channel $[\mathrm{K}(\mathrm{ATP})]$ opener without vasorelaxation: $\mathrm{N}$-(6-aminobenzopyranyl)-N'-benzyl-N' '-cyanoguanidine analogue. J Med Chem 44: 4207-4215, 2001.

7. Yi EY, Park SY, Song HS, et al: KR-31831, a new synthetic anti-ischemic agent, inhibits in vivo and in vitro angiogenesis. Exp Mol Med 38: 502-508, 2006.

8. Folkman J and Haudenschild C: Angiogenesis by capillary endothelial cells in culture. Trans Ophthalmol Soc UK 100: 346-353, 1980.
9. Zachary I: VEGF signalling: integration and multi-tasking in endothelial cell biology. Biochem Soc Trans 31: 1171-1177, 2003.

10. Wu LW, Mayo LD, Dunbar JD, et al: Utilization of distinct signaling pathways by receptors for vascular endothelial cell growth factor and other mitogens in the induction of endothelial cell proliferation. J Biol Chem 275: 5096-5103, 2000.

11. Zakarija A and Soff G: Update on angiogenesis inhibitors. Curr Opin Oncol 17: 578-583, 2005.

12. Los M, Roodhart JM and Voest EE: Target practice: lessons from phase III trials with bevacizumab and vatalanib in the treatment of advanced colorectal cancer. Oncologist 12: 443-450, 2007.

13. Wood JM, Bold G, Buchdunger E, et al: PTK787/ZK 222584, a novel and potent inhibitor of vascular endothelial growth factor receptor tyrosine kinases, impairs vascular endothelial growth factor-induced responses and tumor growth after oral administration. Cancer Res 60: 2178-2189, 2000.

14. Wedge SR, Ogilvie DJ, Dukes M, et al: ZD4190: an orally active inhibitor of vascular endothelial growth factor signaling with broad-spectrum antitumor efficacy. Cancer Res 60: 970-975, 2000.

15. Fong TA, Shawver LK, Sun L, et al: SU5416 is a potent and selective inhibitor of the vascular endothelial growth factor receptor (Flk-1/KDR) that inhibits tyrosine kinase catalysis, tumor vascularization, and growth of multiple tumor types. Cancer Res 59: 99-106, 1999.

16. Fury MG, Zahalsky A, Wong R, et al: A Phase II study of SU5416 in patients with advanced or recurrent head and neck cancers. Invest New Drugs 25: 165-172, 2007.

17. Mita MM, Rowinsky EK, Forero L, et al: A phase II, pharmacokinetic, and biologic study of semaxanib and thalidomide in patients with metastatic melanoma. Cancer Chemother Pharmacol 59: 165-174, 2007.

18. Salzberg M, Pless M, Rochlitz C, Ambrus K, Scigalla P and Herrmann R: A phase I study with oral SU5416 in patients with advanced solid tumors: a drug inducing its clearance. Invest New Drugs 24: 299-304, 2006.

19. Kuenen BC, Tabernero J, Baselga J, et al: Efficacy and toxicity of the angiogenesis inhibitor SU5416 as a single agent in patients with advanced renal cell carcinoma, melanoma, and soft tissue sarcoma. Clin Cancer Res 9: 1648-1655, 2003.

20. Cross MJ, Dixelius J, Matsumoto T and Claesson-Welsh L: VEGF-receptor signal transduction. Trends Biochem Sci 28: 488-494, 2003.

21. Shaheen RM, Davis DW, Liu W, et al: Antiangiogenic therapy targeting the tyrosine kinase receptor for vascular endothelial growth factor receptor inhibits the growth of colon cancer liver metastasis and induces tumor and endothelial cell apoptosis. Cancer Res 59: 5412-5416, 1999. 